\title{
The Impact of Rural Health Care Accessibility on Cancer-Related Behaviors and Outcomes
}

\section{Authors: Eric J. Belasco, Gordon Gong, Barbara Pence, and Ethan Wilkes}

The final publication is available at Springer via http://dx.doi.org/10.1007/s40258-014-0099-4. This is a postprint of an article that originally appeared in Applied Health Economics and Health Policy on June 3, 2014. http://link.springer.com/journal/40258

Belasco, Eric J., Gordon Gong, Barbara Pence, and Ethan Wilkes. "The Impact of Rural Health Care Accessibility on Cancer-Related Behaviors and Outcomes." Applied Health Economics and Health Policy (2014): 1-10.

Made available through Montana State University's ScholarWorks 


\title{
The Impact of Rural Health Care Accessibility on Cancer-Related Behaviors and Outcomes
}

\author{
Eric J. Belasco, Ethan Wilkes; Agricultural Economics \& Economics, Montana State University, P.0. Box 172920, Bozeman, MT \\ 59717-2920, USA
}

Gordon Gong, Barbara Pence; Health Sciences Center, Texas Tech University, Lubbock, TX, USA

\begin{abstract}
Background: This research seeks to identify the relationship between economic factors related to the ability to receive and pay for health services and adverse cancer outcomes, as well as preventative screening and behavioral factors that influence the risk of cancer. We focus on the Northern High Plains region, where we are able to compare regions with extremely low access to health services with those with relatively high levels of access.

Objective: This study aims to identify health disparities in rural communities, particularly among Native American populations, and, thereby, begin to determine the most effective means by which to deliver health services to areas where geography, economics, and culture might prevent traditional models of health delivery from providing sufficient incentives for the prevention of adverse cancer-related outcomes.

Methods: The Health Care Accessibility Index (HCAI) is computed through the use of principal component analysis and includes economic variables as well as variables concerning institutional and geographic access to health care. Index values are then regressed onto cancer outcomes, cancer-prevention outcomes, and cancer-related risk, using weighted least squares and quantile regressions. Results: Counties with relatively poor access to health care (low HCAI) also have statistically (1) lower breast cancer screening rates, (2) higher smoking prevalence, and (4) higher cancer-related mortality rates. Breast cancer screening is found to be especially sensitive to areas of low health accessibility.

Conclusions: Empirical results provide support for policy efforts to increase the accessibility of health care services that are targeted to areas with mammography screening rates, high obesity rates, high smoking prevalence, as well as areas near Native American reservation territories.
\end{abstract}

\section{Key Points for Decision Makers}

Wide disparities exist within the Northern High Plains with regard to areas with moderate and low levels of health care accessibility.

Counties with relatively poor access to health care (a low Health Care Accessibility Index) also have statistically lower breast cancer screening rates, higher smoking prevalence, higher obesity prevalence, and higher cancer-related mortality rates.

Counties with the poorest access to health care services have a higher expected marginal benefit from increases in health care access.
Electronic supplementary material The online version of this article (doi:10.1007/s40258-014-0099-4) contains supplementary material, which is available to authorized users. 


\section{Introduction}

Lifestyle and socioeconomic factors, as well as cancer screening behaviors, are important determinants of latestage cancer incidence rates [1]. The relationship between these determinants and incidence rates is important, in part, because they are the main policy tools in combating cancer. As described by Cutler [2], the four main cancer sites (lung, colorectal, female breast, and prostate) have had decreased US age-adjusted mortality rates since the early 1990s. Cutler attributes these reductions to three main factors: (1) behavioral changes (e.g., reduced smoking); (2) more effective and prevalent screening of breast, colon, and prostate cancers; and (3) more effective chemotherapy and surgery. Of the three factors listed, nearly $60 \%$ of the observed decrease can be attributed to behavioral changes and improved/more prevalent screening.

However, these gains have not been experienced across all demographics. One notable subpopulation that has not experienced the substantial gains in early cancer detection is that of Native Americans [3]. For example, Native American women were the only demographic group that did not experience reductions in cancer mortality rates from 1997 to 2007 [4]. Holm et al. [5] performed a study that focused on North Dakota and found that Native Americans were less likely to meet age-appropriate screening recommendations than samples taken from the USA and North Dakota. A lack of preventative screening for cancer is associated with demographic factors, such as age, education level, and marital status, and is also importantly related to economic variables, such as whether someone is unemployed, has a regular health care provider, has health insurance, and is a non-smoker [6]. Native Americans were also more likely to smoke cigarettes, not engage in leisure time physical activity, and be unemployed. The result of this combination of cancer-related risk factors is that Native American Indians and Alaska Natives are also more likely to have late-stage disease diagnosis than non-Hispanic whites [7].

Past studies focusing on inequalities in health and health care services have utilized concentration indices and curves [8]. Many of these methods are described in Wagstaff and van Doorslaer [9], and essentially include plotting the rank of an economic indicator (such as income) along with the rank of a health indicator. As long as the economic indicator can be ranked unambiguously from low to high socioeconomic status, this method can be used to identify the direction of inequality. However, there are two main problems with the use of concentration curves for the current application, which our approach circumvents. First, a single variable, such as income, does not provide an adequate measure of the ability a household has to pay for health services. For example, two households may receive identical income, but will have very different costs associated with health care services if one of the households is located in an area where they must travel a significantly greater distance in order to access health services. By using an index for access to health care services that includes economic and geographic variables, our proposed approach more comprehensively captures differences in access to health care services. The second limitation of using con-centration curves is that it can only inform regarding rel-ative outcomes in the sample, since it is based entirely on ranked ordering. We are able to relate the variables within the Health Care Accessibility Index (HCAI) directly to cancer-related behaviors and outcomes that can be generalized to other regions.

Grossman [10] first derived the demand conditions for health services under the assumption that health was a "durable good" and showed that the demand for health services is negatively related to wage rate and education. In addition to health services, individual behaviors with respect to health are defined as investments or disinvestments in the commodity "'good health.' More recently, Cutler and Lleras-Muney [11] identified that income, health insurance, family background, and cognitive ability explain nearly $60 \%$ of the relationship between education and health behaviors. Further, Cawley and Ruhm [12] state that higher socioeconomic status, as proxied by educational attainment or family income, is generally correlated with healthier behaviors. For example, high school dropouts are more likely to smoke, be obese, drink heavily, and be physically inactive, when compared to college graduates. In the present study, these are important risk behaviors since they all increase the likelihood of cancer. Even modest marginal changes in cancer mortality rates, through diminishing the presented risk factors, can have significant impacts, as shown by Murphy and Topel [13], who estimated that a $1 \%$ reduction in the cancer mortality rate would be worth approximately US $\$ 500$ billion.

Additional research has evaluated the linkage between insurance participation and health outcomes. However, Kenkel [14] points out that insurance expansion alone does not lead to substantial improvements in preventative services, which may be due to the other costs, such as lack of convenience, that provide important barriers to access to preventative care. While the cited papers provide valuable insights in linking the consumption of medical services and economic variables, they lack an approach that accounts for the many facets of the ability a household has to con-sume and pay for health care services. This implies that these studies do not consider the full cost of preventative care. For example, many studies often do not include important cost factors such as the distance to care facility, supply of local physicians, health care coverage, among other important economic variables. This research seeks to broaden the definition of economic factors that influence the demand for health care to include travel costs, the supply of nearby physicians, as well as an array of eco-nomic factors that contribute to an individual's ability to attend and pay for 
attend and pay for medical services. This research seeks to identify the relationship between these economic factors and the main drivers of adverse cancer outcomes, which include preventative screening behavior and behavioral factors that increase the risk of cancer.

Adverse cancer-related outcomes, such as increased late-stage cancer detection and cancer-related mortalities, are linked to lower preventative screening rates, which occur with more regularity in areas where a higher effective cost and often a decreased ability to pay for health care services are experienced. Cancer-related risk behaviors, such as smoking and obesity, might also be linked to the availability and ability to pay for health care ser vices when one considers the importance of access to smoking cessation and weight loss or activity enhancement programs, which are likely to be correlated wit $\mathrm{h}$ other complementary health-related services.

In focusing on the Northern High Plains region of the USA, we are able to compare counties with extremely low access to health services with those with relatively high levels of access. The findings from this research are crucial in identifying health disparities in rural communities, particularly in communities containing Native American populations. Additionally, the results provide insight to help determine the most effective means by which to deliver health services to areas where geography, economics, and culture might prevent traditional models of health delivery from providing sufficient incentives for the prevention of adverse cancer-related outcomes.

\section{Empirical Methods}

\subsection{Data}

Data from 2005 to 2009 were collected for county-level, age-adjusted, all-site, late-stage incidence, total incidence, and cancer-related mortality rates. These data were averaged across the given time period in order to comply with state regulations with regard to patient confidentiality, and were provided from the state National Program of Cancer Registries for Montana, Wyoming, North Dakota, and South Dakota. In addition to data confidentiality issues, annual incident and mortality estimates provided for rela-tively small counties often lack an appropriate degree of precision. By aggregating across 5 years and creating of a crosssection of data, the variables used in this study are believed to provide a more precise estimate of incident and mortality rates while complying with state confidentially regulations. Late-stage incidence cancers include all-site cancers that were diagnosed at 'regional' or 'distant' stages. Data on mammography prevalence in the last 2 years and cancer-related risk behaviors (current smoking prevalence, obesity prevalence) are from 'Model-Based Small Area
Estimates of Cancer Risk Factors \& Screening Behaviors' (http://www.sae.cancer.gov) that provide county-level estimates aggregated over 2000-2003 based on the methodology in Raghunathan et al. [15]. Using these years provides an indicator prior to observing cancer incidence and mortalities, and is the most recent countylevel estimate.These county-level data are estimated using a hierarchical Bayesian procedure that incorporates data from the Behavioral Risk Factor Surveillance System (BRFSS) and the National Health Interview Survey (NHIS). The approach described in Raghunathan et al. [15] corrects for non-coverage bias and nonresponse bias in BRFSS data, while reducing the variability associated with small samples. Zhang et al. [16] evaluated the usefulness of the data used in this study and concluded that the value was especially high when estimating cancer screening rates in rural areas.

Economic data are also used in this study to compute the HCAI, and are from the US Census Bureau's American Community Survey (ACS), 2010 Census, and BRFSS. Data from the ACS include variables included in many socioeconomic indices of socio-economic well-being [17, 18] $]^{1}$.

In addition to the variables used by Albrecht and Ramasubramanian [17], we include additional economic variables such as the percentage of residents without health

insurance and mean household income and benefits. Estimates for the prevalence of uninsured in each county are averages of annual data from 2005 to 2009 from the Census Bureau's Small Area Health Insurance Estimates program. Calculated from the center of each county to the nearest cancer care facility accredited by the Commission on Cancer of the American College of Surgeons. Facilities that were accredited in 2008 or later were excluded. The log of the number of general practice medical doctors (MDs) was obtained from the BRFSS and includes general-practice and family-medicine, patient-care, office-based, non-federal MDs per 100,000 people in 2006.

These variables are utilized in this study in order to assess the cost of medical care services related to cancer prevention and detection. For example, the ability to pay for medical services is largely influenced by economic circumstances, which are explained with the use of employment and economic independence variables. In addition to income and other economic factors, the ability of an individual to receive medical services is largely

\footnotetext{
1 Albrecht and Ramasubramanian [17] developed an index of social well-being using the following ten variables: the percentage of residents (1) receiving any form of income support; (2) with any form of disability; (3) not living in own home; (4) living in a home with too few bedrooms; (5) without a high school diploma or equivalent; (6) in single-parent households; (7) below the poverty level; (8) without a car; (9) who were unemployed; and (10) without a phone.
} 
influenced by the supply of services. For this reason, medical supply variables are included that also include the distance to cancer-care facilities. This is particularly important when we consider rural communities where the economic cost of medical care may come in the form of travel costs to care facilities due to a lack of local physicians or expertise.

\subsection{Principal Component Analysis}

In order to aggregate the impact of each of these variables, and accounting for the relatively small sample size, the HCAI is computed through the use of principal component analysis. Principal component analysis is used to take a set of variables and find a linear combination of those vari-ables that can be utilized to capture the explained vari-ability between variables [19]. This effectively reduces the number of estimable variables, which is particularly important in situations where each variable has new information but is highly correlated with many of the other variables. When presented with a regression that contains high degrees of multicollinearity, marginal effects are often difficult to isolate, resulting in high standard errors. For this application, we assume that the HCAI is a linear function

of the included variables $(z)$, such that HCAI $=a^{\prime} z+e$, where $z$ includes variables linearly related to the $H C A I$ through the first principal component (a) [ 20]. Belasco et al. [18] used county-level data from Texas to demon-strate the use of fitted health accessibility index values to partially explain cancer-related outcomes, and this resulted in superior goodness-of-fit based on Akaike's Information Criteria and Schwartz Information Criteria. We also include the flexibility parameter $(1 / a)$, which provides a measure of which $z$ needs to change in order to observe a 1-unit change in HCAI.

The first principal component is typically used to develop the index. In this application, nearly $50 \%$ of the variation in these variables is explained through the first principal component. The flexibility parameter is important as it documents the amount $z$ would need to change in order to expect a 1-unit change in HCAI and allows us to com-pare the degree to which changes in the components of the index would need to change in order to change the index. This provides insight into the relative importance of each variable in the index and translates the marginal effect from the HCAI onto its input variables. One difficulty with this particular approach is that given that elements in $z$ are highly correlated, it is difficult to imagine an exogenous shock that only impacts one element of $z$ without influ-encing the others. That being said, the results present the scale necessary in order to change the HCAI. For example, the percentage of residents who are under the poverty level has a negative impact on the HCAI. In order to increase the HCAI by 1 unit, the percentage under the poverty level would need to decrease by 1.80 percentage points. Along with poverty rate, income appears to have a strong impact on the HCAI, as an average household income increase of US $\$ 1,860$ within a county is associated with a 1-unit increase in the HCAI. More details can be found in the supplemental appendix.

To illustrate the graphical nature of the estimated HCAI values, Fig. 1 displays a plot of HCAI values by county. Dots within the figure are set to emphasize the location of counties that have Native American populations of higher than $40 \%$ of total residents. The close relationship in this region between extremely poor health access and Native American population density is striking. This is especially notable in the north-central and south-central regions of South Dakota, which include the Oglala and Rosebud Sioux tribal regions and have some of the lowest HCAI values in our sample. Montana, as well, shows particularly low index values in locations in and around the Crow Nation, Fort Peck, and Blackfeet reservation territories. It is also worth mentioning the negative skewness associated with HCAI values indicating a great numeric distance between counties found in the lowest 10 th percentile and the rest of the sample, as shown in Fig. 2.

\subsection{Regression}

Table 1 presents summary statistics for the associated independent and dependent variables used in this study for the counties included in this data. Weighted mean statistics are weighted by 2010 population in order to more accurately represent the region as a whole and not bias results with respect to the impact of counties with relatively small populations. In addition to the weighted means, 10th, 50th, and 90th percentiles are presented to characterize distributional characteristics and identify the spread in out-comes. For example, the age-adjusted, all-site, cancer-related mortality rates increase by $61 \%$, from 131.92 to 212.82 per 100,000 residents, when moving from the 10th to 90th percentile. The percentage of late-stage cancer incidence also increases by $52 \%$ when moving from the 10th to 90 th percentile. Changes in incidence and mortality rates are hypothesized to be partially influenced by screening and participation in risky behaviors. When comparing the 10th and 90th percentiles, we also view substantial differences in mammography screening rates (57 vs. $71 \%$ ), smoking prevalence (18 vs. $28 \%$ ), and obesity prevalence ( 23 vs. 31 $\%$ ). Each of these variations are larger than $25 \%$, with the largest distinction being in smoking rate, which presents a $58 \%$ increase between the 10th and 90th percentile.

Distance to cancer care and the supply of local physicians are also hypothesized to be importantly related to the ability a consumer of health services has to detect and effectively treat cancer prior to late-stage cancer phases. The 10th percentile for medical supply indicates $2.00 \mathrm{MDs}$ 
Fig. 1 2005-2009 Health Care Accessibility Index (HCAI) values, by county, for Montana (MT), Wyoming (WY), North Dakota (ND), and South Dakota (SD). Blue circles indicate over 40 $\%$ of the population was classified as Native American by the 2010 Census

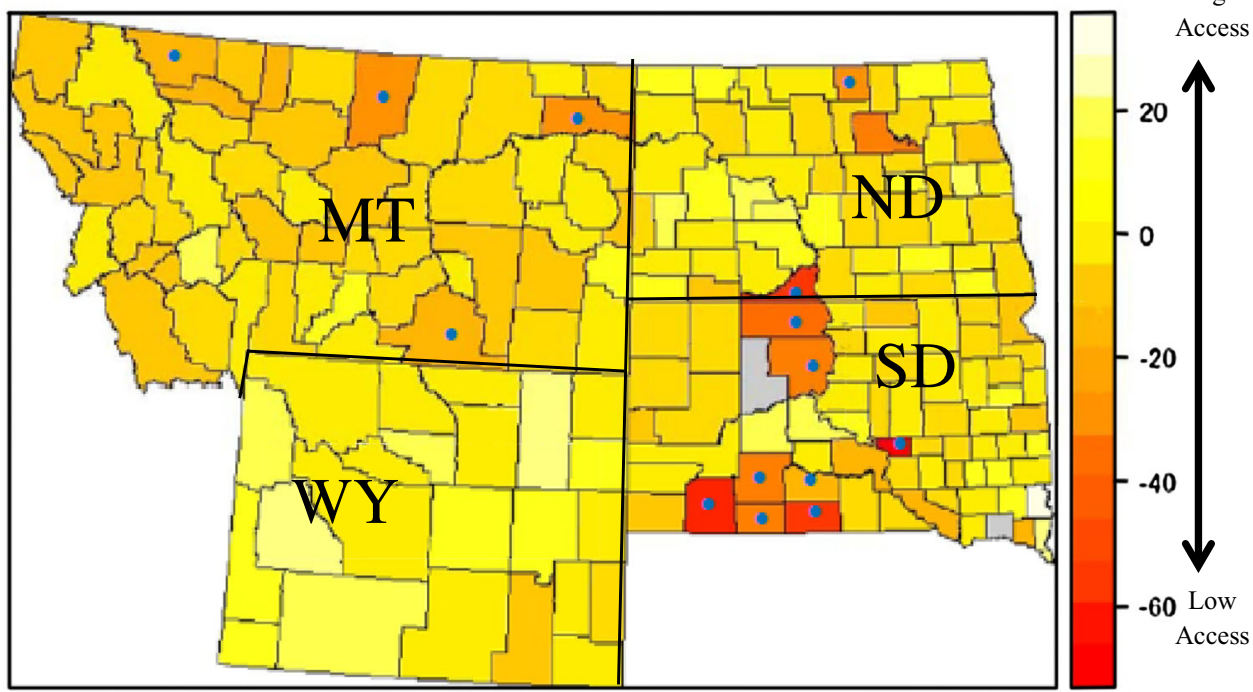

Indicates over $40 \%$ of population classified as Native American by 2010 Census

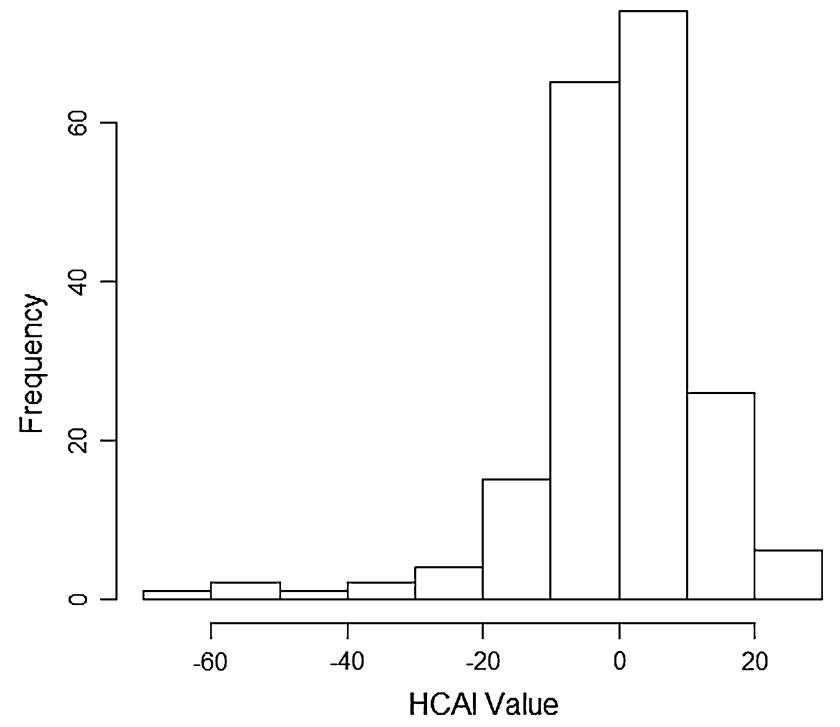

Fig. 2 Empirical distribution of county-level Health Care Accessibility Index (HCAI) values

per 100,000 residents. This lack of local supply implies that travel is often necessary for even basic medical services. Further, $10 \%$ of the population is at least 158.79 miles away from an accredited cancer care facility. While many of the included economic variables present wide variation within our sample, the percentage below the poverty level is especially notable, as moving from the 10th to 90th percentile has this value change from 8 to $23 \%$, which is a $188 \%$ increase. Additionally, this sample provides a relatively stark contrast with regard to education, as counties in the 90th percentile have $20.21 \%$ of the popul- ation over the age of 18 years without a high school or equivalent education level. This is $136 \%$ higher than the value for the lower 10th percentile within this region (8.56 $\%)$. These distinctions are presented in order to provide support for using this particular data sample, where large variations between important indicators of cancerrelated outcomes and behaviors are present. These indicators are shown to include economic, education, health screening, risky behaviors, and health supply variables.

In order to present the differences in dependent variable levels for different HCAI levels, Table 2 splits weighted means by tercile rankings and lowest decile rankings for HCAI. Recall that low HCAI levels indicate regions with relatively low levels of access to health care services for cancer. These indicators present a series of challenges for counties faced with low HCAI. For example, both obesity and smoking prevalence are associated with increased cancer risk. Additionally, lower mammography rates also present challenges in combating cancer in these areas. Breast cancer mortality rates have dropped substantially over the years and are largely attributed to better screening rates from mammography [2]. The percentage of the population that is Native American is also notably higher in counties within the lowest HCAI tercile and decile. In many of these areas, the Indian Health Services (IHS) covers preventative screening for breast cancer [21]. However, economic costs for medical services might also include substantial travel costs in rural areas. Each of these movements are magnified in the lowest decile as ageadjusted mortality rates, smoking prevalence, obesity 
Table 1 Principal component analysis results from variables in Health Care Accessibility Index (HCAI) and weighted summary statistics

\begin{tabular}{|c|c|c|c|c|c|c|}
\hline \multirow[t]{2}{*}{ Variable } & \multirow{2}{*}{$\begin{array}{l}\text { First principal } \\
\text { component score }\end{array}$} & \multirow{2}{*}{$\begin{array}{l}\text { Flexibility } \\
\text { parameter }\end{array}$} & \multirow{2}{*}{$\begin{array}{l}\text { Weighted } \\
\text { mean }\end{array}$} & \multicolumn{3}{|c|}{ Percentiles } \\
\hline & & & & $10 \%$ & $50 \%$ & $90 \%$ \\
\hline \multicolumn{7}{|l|}{ Dependent variables } \\
\hline Percentage of late-stage cancer incidence $(\%)$ & & & 41.62 & 33.13 & 42.11 & 50.32 \\
\hline $\begin{array}{l}\text { Age-adjusted cancer-related mortality rate (deaths per } \\
100,000 \text { residents) }\end{array}$ & & & 170.46 & 131.92 & 171.90 & 212.82 \\
\hline Mammography within last 2 years (\%) & & & 67.33 & 57.05 & 64.65 & 71.45 \\
\hline Current smoking prevalence $(\%)$ & & & 23.29 & 17.85 & 22.90 & 28.20 \\
\hline Obesity prevalence $(\%)$ & & & 25.58 & 22.65 & 27.60 & 31.15 \\
\hline \multicolumn{7}{|l|}{ Independent variables } \\
\hline HCAI & & & 1.36 & -13.37 & 1.70 & 12.03 \\
\hline Distance to accredited cancer care facility (miles) & -0.02 & -57.62 & 95.81 & 29.04 & 75.17 & 158.79 \\
\hline Mean household income and benefits (in US $\$ 1,000 \mathrm{~s}$ ) & 0.54 & 1.86 & 54.90 & 44.47 & 52.56 & 64.92 \\
\hline Proportion of single-parent households (\%) & -0.41 & -2.45 & 11.44 & 6.05 & 10.36 & 17.77 \\
\hline Proportion of households receiving assistance (\%) & -0.25 & -3.99 & 36.07 & 28.34 & 38.16 & 47.37 \\
\hline Proportion of households with no vehicle available (\%) & -0.14 & -7.22 & 4.38 & 1.95 & 4.15 & 7.60 \\
\hline Proportion of housing units renter occupied (\%) & -0.46 & -2.16 & 28.93 & 18.45 & 26.35 & 37.30 \\
\hline $\begin{array}{l}\text { Proportion of households with less than one room per } \\
\text { occupant }(\%)\end{array}$ & -0.17 & -5.83 & 2.34 & 0.10 & 1.20 & 4.10 \\
\hline $\begin{array}{l}\text { Proportion of households with no telephone service } \\
\text { available }(\%)\end{array}$ & -0.10 & -10.51 & 3.16 & 0.90 & 3.00 & 6.75 \\
\hline Proportion of civilian labor force unemployed (\%) & -0.29 & -3.45 & 5.06 & 1.50 & 3.30 & 8.45 \\
\hline $\begin{array}{l}\text { Logged medical specialist medical doctors per } 100,000 \\
\text { population }\end{array}$ & 0.01 & 86.81 & 5.42 & 0.69 & 7.19 & 7.60 \\
\hline Proportion of people under poverty level (\%) & -0.56 & -1.80 & 13.73 & 7.80 & 12.30 & 22.65 \\
\hline Proportion uninsured (\%) & -0.05 & -20.02 & 19.25 & 12.59 & 18.21 & 25.68 \\
\hline $\begin{array}{l}\text { Proportion of population over the age of } 18 \text { years with less } \\
\text { than high school or equivalent }(\%)\end{array}$ & -0.20 & -4.88 & 12.70 & 8.56 & 13.30 & 20.21 \\
\hline
\end{tabular}

Table 2 Weighted mean statistics, by Health Care Accessibility Index (HCAI) quantile ranking

\begin{tabular}{|c|c|c|c|c|}
\hline \multirow[t]{2}{*}{ Variable } & \multirow{2}{*}{$\begin{array}{l}\text { Decile } \\
\text { Lowest }\end{array}$} & \multicolumn{3}{|l|}{ Tercile } \\
\hline & & Lowest & Middle & Highest \\
\hline Late-stage cancer incidence $(\%)$ & 44.75 & 40.49 & 41.92 & 42.24 \\
\hline Age-adjusted cancer-related mortality rate (deaths per 100,000 residents) & 191.13 & 179.99 & 165.33 & 167.53 \\
\hline Mammography within last 2 years $(\%)$ & 55.89 & 65.30 & 67.82 & 68.47 \\
\hline Current smoking prevalence $(\%)$ & 29.31 & 24.62 & 22.75 & 22.72 \\
\hline Obesity prevalence $(\%)$ & 33.68 & 26.41 & 25.32 & 25.16 \\
\hline Percentage of total population Native American (\%) & 53.29 & 13.43 & 3.62 & 1.55 \\
\hline
\end{tabular}

prevalence, and the percentage of total population that is Native American are substantially higher, while mammography screening rates are substantially lower.

The computed index provides a single metric that can be used to evaluate and rank access to health care accessibility in order to compare counties within our sample. The index is then regressed onto cancer outcomes (percentage of late- stage cancer incidence, relative to all cancer incidence), cancer prevention outcomes (percentage of population that receives appropriately recommended breast cancer screenings), and cancer-related risk behaviors (percentage of the population that smokes cigarettes and percentage that is classified as obese), using a weighted regression. Each regression is weighted by the 2010 population in 
order to provide estimates that are representative of the four-state region. County-level regressions are used to identify correlations between outcome variables and the index.

Quantile regressions are also used in order to assess the potentially different impacts of the HCAI on counties with a range of HCAI values. Quantile regressions allow for marginal effects to be evaluated across the range of defined quantiles in order to identify the existence of a changing relationship between the HCAI and dependent variables of interest. This is particularly useful in this study as it allows for a richer characterization of the heterogeneity [22]. Additionally, quantile regressions are less sensitive to outliers and are estimated consistently under fairly mild assumptions [23].

\section{Results}

Weighted regression results are provided in Table 3 and indicate the relationships between important dependent variables and HCAI values. In general, counties in our sample with relatively poor access to health care have (1) a lower prevalence of breast cancer screening, (2) higher prevalence of risk-related behaviors such as obesity and smoking, and (3) higher rates of cancer-related mortalities. It is interesting to note that there does not appear to be a statistically significant relation- ship between health care accessibility and late-stage cancer incidence.

While low cancer screening rates and high prevalence of cancer-related behaviors, such as obesity and smoking, are known to contribute to cancer incidence and mortality, it is difficult to identify which of these aspects are more problematic in areas of low access because of the magnitude of both problem areas. For example, areas with dense Native American populations in our sample also tend to have high obesity rates, high smoking rates, low breast cancer screening rates, and low access scores.

As suggested in Solon et al. [24], weighted results can be useful in obtaining average partial effects but typically demand additional analysis in order to identify the heterogeneity within the sample. For this reason, weighted regressions were split into two subsamples in Table 3 to illustrate the differences between densely populated Native American regions and other regions. This distinction is drawn to characterize any differences between an IHSserviced community and a community with a more traditional health care model. IHS-serviced areas are proxied as counties with a higher than $40 \%$ population that is classified as Native American by the 2010 Census. The relationships between the HCAI and the prevalence of mammography screening, smoking, and obesity are all heightened when only densely populated Native American counties are considered. When we consider the remaining

Table 3 Weighted least squares regression results

\begin{tabular}{|c|c|c|c|c|c|c|}
\hline \multirow[t]{3}{*}{ Sample } & \multirow[t]{3}{*}{ Dependant variable } & \multicolumn{5}{|c|}{ Independent variable } \\
\hline & & \multicolumn{2}{|l|}{ Constant } & \multicolumn{3}{|l|}{ HCAI } \\
\hline & & Est. & SE & Est. & SE & $P$ value \\
\hline \multirow[t]{5}{*}{ All Obs $(n=196)$} & Mammography within last 2 years & $66.97 *$ & 0.42 & $0.26 *$ & 0.04 & $<0.01$ \\
\hline & Current smoking prevalence & $23.46^{*}$ & 0.21 & $-0.13^{*}$ & 0.02 & $<0.01$ \\
\hline & Obesity prevalence & $25.75^{*}$ & 0.27 & $-0.13 *$ & 0.03 & $<0.01$ \\
\hline & Percentage of late-stage cancer incidence & $41.64 *$ & 0.47 & -0.02 & 0.04 & 0.69 \\
\hline & Age-adjusted cancer-related mortality rate & $171.06^{*}$ & 1.73 & $-0.44 *$ & 0.16 & $<0.01$ \\
\hline \multirow[t]{5}{*}{ Dense NA/AI counties $(n=14)$} & Mammography within last 2 years & $67.29 *$ & 2.79 & $0.51 *$ & 0.08 & $<0.01$ \\
\hline & Current smoking prevalence & $25.93 *$ & 1.49 & $-0.20 *$ & 0.04 & $<0.01$ \\
\hline & Obesity prevalence & $30.94 *$ & 1.41 & $-0.14 *$ & 0.04 & $<0.01$ \\
\hline & Percentage of late-stage cancer incidence & $43.39 *$ & 3.66 & -0.04 & 0.11 & 0.70 \\
\hline & Age-adjusted cancer-related mortality rate & $227.02 *$ & 17.62 & 1.13 & 0.53 & 0.05 \\
\hline \multirow[t]{5}{*}{ Remaining counties $(n=182)$} & Mammography within last 2 years & $67.71^{*}$ & 0.42 & 0.08 & 0.05 & 0.08 \\
\hline & Current smoking prevalence & $23.05^{*}$ & 0.20 & -0.04 & 0.02 & 0.10 \\
\hline & Obesity prevalence & $25.25^{*}$ & 0.27 & -0.02 & 0.03 & 0.61 \\
\hline & Percentage of late-stage cancer incidence & $41.41^{*}$ & 0.51 & 0.03 & 0.06 & 0.58 \\
\hline & Age-adjusted cancer-related mortality rate & $170.77 *$ & 1.82 & -0.47 & 0.21 & 0.03 \\
\hline
\end{tabular}

'Dense NA/AI counties' include counties where over $40 \%$ of the population was classified as Native American by the 2010 Census. 'Remaining counties' include all other counties

Est. estimate, HCAI Health Care Accessibility Index, NA/AI Native American/American Indian, Obs observations, SE standard error

* Denotes that parameter estimate is statistically significant at 0.01 significance level 

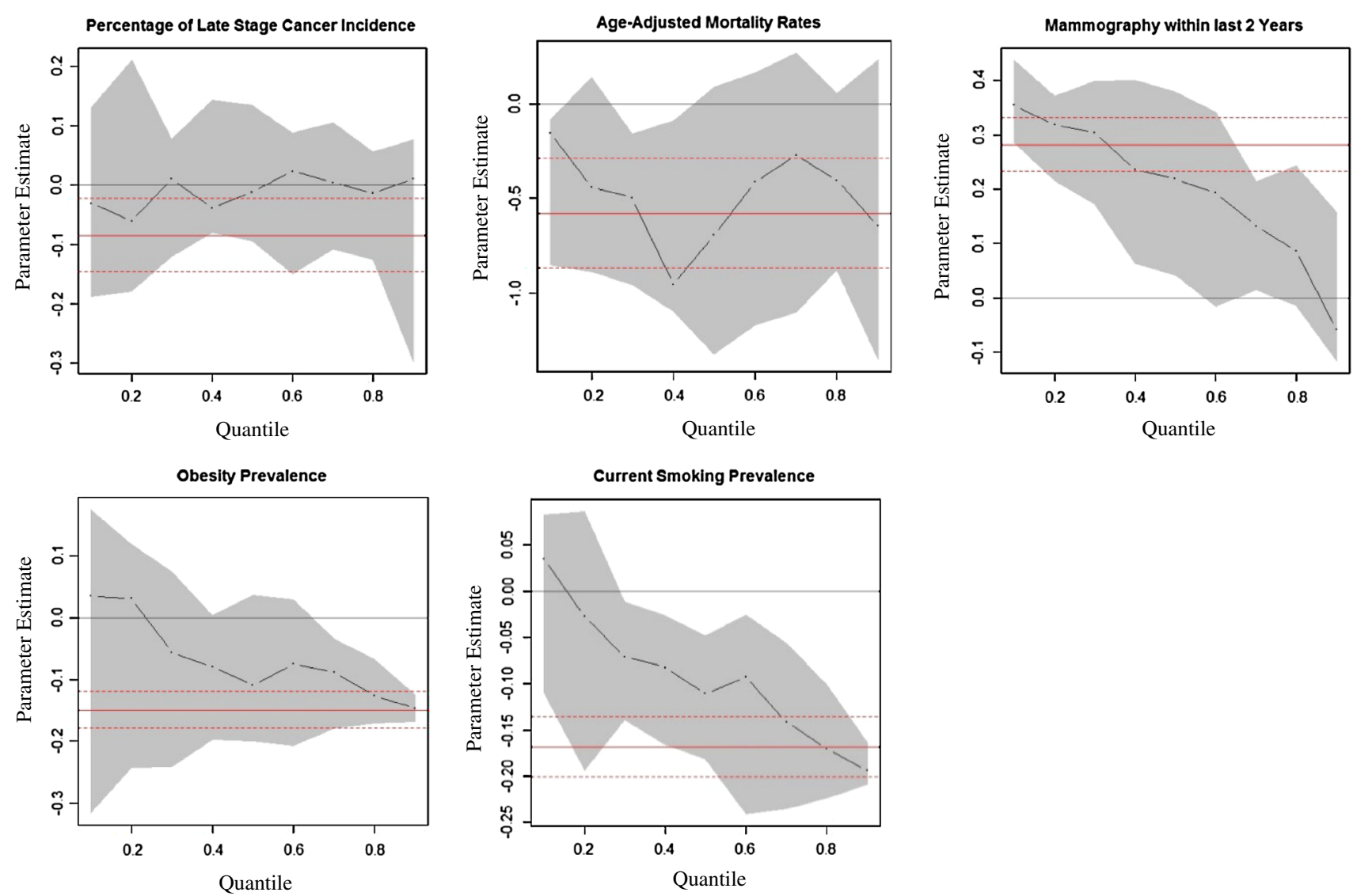

Fig. 3 Plot of quantile regression results for marginal impact of Health Care Accessibility Index (HCAI) values on major indicators of cancer-related outcomes. Note, black lines indicate weighted quantile regression results, with grey areas indicating confidence intervals. Solid and dotted red lines indicate weighted ordinary least squares parameter estimates and confidence intervals, respectively counties separately, much of the statistical significance disappears, which may be due to the relative lack of variation in the HCAI for the remaining counties. These results highlight the heightened importance of improvements to health care access, especially in those areas where traditional models of health care delivery are not utilized.

Figure 3 presents a plot of the weighted quantile results with respect to the marginal relationships between the HCAI and major indicators, across the range of quantiles with regard to indicators. This allows for a more detailed assessment of the marginal relationship between access to the health care services and cancer-related behaviors and outcomes by isolating the impacts for a range of access to health care services. For example, age-adjusted mortality rates and the percentage of metastatic cancer cases are shown to be not statistically different from zero when controlling for quantile. This is in con-trast to ordinary least squares (OLS) results which indi-cate statistically significant results. The sub-plot regarding appropriately timed mammography indicates that counties with the lowest mammography screening rates are asso-ciated with large increases in screening rates for relatively mild gains in accessibility. As shown, the counties with the lowest screening rates (lowest quantiles) are shown to have a statistically significant positive relationship, implying that advances in health access can have rela-tively large gains in the prevalence of mammography screening. The lowest quantile has a marginal impact that is slightly larger than the marginal impact estimated from OLS. However, OLS estimates provide a single estimate, while the quantile results indicate that this marginal impact is not statistically significant for counties with relatively high screening rates (top 3 deciles). Results regarding the prevalence of cervical cancer screening are somewhat ambiguous, with a positive relationship between mid-level screening counties and an insignificant relationship for the top and bottom counties with respect to screening.

The results with regard to obesity prevalence provide a statistically insignificant relationship between access and obesity among lower deciles. It is worth mentioning that the variance within this lowest tier group is substantially wide and shrinks in higher quantiles. For counties with relatively high levels of obesity (top 3 quantiles), there is a statistically significant negative relationship indicating higher obesity rates with low levels of access. 


\section{Discussion}

While both prevention and risk-related behaviors are known to impact the early detection of many cancers, this research identifies the rather complex web of economic issues that impacts less desirable cancer outcomes. In order to reduce the cancer burden, it is crucial that (1) cancerrelated prevention activities are expanded and (2) riskrelated behaviors are mitigated. However, counties in our sample that have poor access to health care are adversely faced with low levels of cancer prevention participation and high levels of risk-related behaviors (i.e., obesity and smoking). Future research is needed in order to focus on isolating the impact of screening and risk-related behaviors on cancer outcomes so that federal efforts and grants can be more efficiently focused on crucial populations. In a time of belt tightening for federal funds, it will be crucial for research and prevention programs to be as cost-effective as possible.

Results in this research indicate the largest potential gains in preventing cancers can be found in areas with low screening rates, high obesity rates, and high smoking rates. Quantile regression results demonstrate the largest marginal effects from changes in the HCAI can be found in areas with the lowest screening rates and highest risk factors (e.g., smoking and obesity). Areas that contain the lowest accessibility scores, in terms of the HCAI, are often present in and around counties that have a relatively high population of Native Americans. These regions also tend to be the most remote areas in our sample.

While not a regressor in our model specifications, the percentage of Native American population tends to be highly correlated with adverse cancer outcomes and behaviors in our sample. For example, counties in our sample with a high proportion of Native American residents also tend to be characterized with high obesity rates, high smoking rates, and low cancer screening rates. This paper posits that late-stage cancer detection can be explained using economic factors that characterize the cost and ability to cover health care services. This interpretation is consistent with that of Belasco et al. [18], who evaluated health disparities in Texas, where Hispanic populations tended to have higher late-stage cancer detection rates while also lacking access to health care services.
While there may be other factors that drive higher rates among Native American populations related to culture, genetics, or unique health delivery systems (i.e., the IHS), the variables used in this study provide policy instruments by which to influence health outcomes. For example, improvements in the HCAI come from a list of variables that can be implemented into policy, such as the supply of physicians, distance to cancer care facilities, unemployment, the percentage of residents below the poverty level, and the percentage of uninsured. Given the negative skewness in HCAI (shown in Fig. 2), it is apparent that counties in the lower tail of the distribution need to make significant improvements in the included economic variables in order to realize gains in cancer-related outcomes and behaviors.

There are also a few notable limitations of this study. First, while incident rates present an important metric in cancer outcomes, it is also a somewhat biased measure. This is due to the fact that incident rates only include known cases, while cancer is often latent for some time before being detected. Second, isolating the effect of a single policy is challenging given the highly correlated nature of the dependent variables used in this study, particularly in counties that reside in the lower tail of the HCAI. This presents the question as to what is the best policy to combat cancer. However, health behaviors and screening rates are shown to move together, implying that these either reinforce one another or are highly related.

Funding and conflict of interest No external funding was received for this paper. The authors have no conflicts of interest to report.

Author contribution The paper was conceived by EB and BP. Major contributions to the 'Empirical Methods' sections were made by EB, EW, and GG. Primary contributions by EW included data collection and statistical analysis, while GG primarily contributed in the implementation of the principal component analysis and its interpretations. Major contributions to the 'Introduction' and 'Discussion' sections were made by EB and BP. EB is the guarantor for the overall content. 


\section{References}

1. American Cancer Society. Cancer facts and figures 2013. 2013. http://www.cancer.org/research/cancerfactsfigures/cancerfacts figures/cancer-facts-figures-2013.

2. Cutler DM. Are we finally winning the war on cancer? J Econ Perspect. 2008;22(4):3-26.

3. Cobb N, Wingo PA, Edwards BK. Introduction to the supplement on cancer in the American Indian and Alaska native populations in the United States. Cancer. 2008;113: 1113-16. doi:10.1002/ cncr.23729. Accessed 16 May 2012.

4. Siegel R, Ward E, Brawley O, Jemal A. The impact of eliminating socioeconomic and racial disparities on premature cancer deaths. CA A Cancer J Clin. 2011;61:212-36. doi:10.3322/caac. 20121. Accessed 16 May 2012.

5. Holm JE, Vogeltanz-Holm N, Poltavski D, McDonald L. Assessing health status, behavioral risks, and health disparities in American Indians living on the Northern Plains of the US. Publ Health Rep. 2010;125:68-78.

6. Muus KJ, Baker-Demaray T, McDonald LR, Ludtke RL, Allery AJ, Bogart TA, Goldberg J, Ramsey SD, Buchwald DS. Body mass index and cancer screening in older American Indian and Alaska native men. J Rural Health. 2009;25(1):104-8.

7. Perdue DG, Perkins C, Jackson-Thompson J, Coughlin SS, Ahmed F, Haverkamp DS, Jim MA. Regional differences in colorectal cancer incidence stage, and subsite among American Indians and Alaska natives, 1999-2004. Cancer. 2008;113:1179-90. doi:10.1002/cncr.23726. Accessed 16 May 2012.

8. Fleurbaey M, Schokkaert E. Equity in health and health care. In: Pauly MV, Mcguire TG, Barros PP, editors. Handbook Health Econ, vol. 2. Elsevier: Amsterdam; 2012. p. 1003-1092.

9. Wagstaff A, van Doorslaer E. Equity in health care finance and delivery. In: Culyer A, Newhouse J, editors. Handbook of health economics, vol. 1B. Elsevier: Amsterdam; 200. p. 1803-1862.

10. Grossman M. On the concept of health capital and the demand for health. J Polit Econ. 1972;80(2):223-55.

11. Cutler DM, Lleras-Muney A. Understanding differences in health behaviors by education. J Health Econ. 2010;29:1-28.

12. Cawley J, Ruhm CJ. The economics of risky health behaviors. In: Pauly MV, Mcguire TG, Barros PP, editors. Handbook of health economics, vol. 2. Elsevier: Amsterdam; 2012. p. 45-93.
13. Murphy KM, Topel RH. The value of health and longevity. J Polit Econ. 2006;114(5):871-904.

14. Kenkel D. Prevention. In: Cuyler AJ, Newhouse JP, editors. Handbook of health economics, vol. 1. Elsevier: Amsterdam; 2000. p. $1675-720$.

15. Raghunathan TE, Xie D, Schenker N, Parsons V, Davis WW, Dodd KW, Feuer EJ. Combining information from two surveys to estimate county level prevalence rates of cancer risk factors and screening. J Am Stat Assoc. 2007;102(478):474-86.

16. Zhang Y, Jones B, Rahman RL, Johnson L, Jenkins M, Pence B. Challenges associated with assessment and use of mammography screening rates in rural West Texas. Texas Publ Health J. 2012;64(2):20-4.

17. Albrecht J, Ramasubramanian L. The moving target: a geographic index of relative wellbeing. $J$ Med Syst. 2004;28(4):371-84.

18. Belasco EJ, Phillips BU Jr., Gong G. The Health Care Access Index as a determinant of delayed cancer detection through principal component analysis. In: Sanguansat P, editor. Principal component analysis-multidisciplinary approaches. Rijeka: InTech; 2012. p. 143-166.

19. Phillips BU Jr, Gong G, Hargrave KA, Belasco E, Lyford CP. Correlation of the ratio of metastatic to non-metastatic cancer cases with the degree of socioeconomic deprivation among Texas counties. Int J Health Geograph. 2011;10:12.

20. Greene WH. Econometric analysis. 7th ed. Upper Saddle River: Prentice Hall; 2011.

21. Haverkamp Donald, Perdue David G, Espey David, Cobb Nathaniel. A survey of Indian Health Service and tribal health providers' colorectal cancer screening knowledge, perceptions, and practices. J Health Care Poor Underserved. 2011;22:243-57.

22. Koenker R, Hallock F. Quantile regression. J Econ Perspect. 2001;15:143-56.

23. Cameron AC, Trivedi PK. Microeconometrics: methods and applications. New York: Cambridge University Press; 2005.

24. Solon G, Haider SJ, Wooldridge J. What are we weighting for? NBER working paper no. 18859. 2013. http://www.nber.org/ papers/w18859. 